\title{
Microbial Adaptation to Bromobenzene in a Chemostat
}

\author{
George T. Sperl and Gregory J. Harvey \\ Department of Natural Sciences, University of Michigan-Dearborn, Dearborn, Michigan, USA
}

\begin{abstract}
Microorganisms capable of growth with bromobenzene as their sole source of carbon and energy were selected from a continuously growing community of microorganisms initially selected for growth at the expense of benzene as sole source of carbon and energy. The selection was by a gradient of increasing bromobenzene/decreasing benzene in the nutrient feed of the continuous culture. Generation times in the system were about $38 \mathrm{~h}$. These experiments selected two strains of Pseudomonas that used bromobenzene as sole carbon and energy source. These two strains appeared identical with two members of the original community which previously could not use bromobenzene. After the selection, the strains in either pure or mixed culture, were capable of growing at the expense of benzene, bromobenzene, and chlorobenzene, but not iodobenzene.
\end{abstract}

The degradation of man-made, nonnatural (xenobiotic), potentially toxic chemicals in the environment can follow two different metabolic paths: (a) co-metabolism with other organic materials, which is a piecemeal, stepwise process in which several different microorganisms are usually involved; and (b) complete mineralization of the chemical by one particular organism as its sole source of carbon and energy. If the xenobiotic chemical is toxic, then the simple disappearance of the chemical does not necessarily imply its detoxification, unless it is completely mineralized. Co-metabolism is of considerable ecological importance since co-metabolic attack on xenobiotic chemicals probably occurs frequently in nature $[7,8,9]$ and is probably the major degradative path. Co-metabolism can require the presence of several species for complete mineralization, or it may mean that a certain chemical is only partially metabolized. With this path, a chemical that might pose an in soluble metabolic problem to a single species may be successfully attacked catabolically by a community of different microorganisms and be completely mineralized [13]. The concept of co-metabolism also allows the possibility of detoxification by incomplete degradation. The difficulty with this outcome is that the resultant metabolic product may also be toxic [10].

Therefore, if one wishes to be sure that a chemical is completely broken down and detoxified, pure cultures or mixed cultures growing at the expense of only one compound would give superior results. There are fewer variables with this work, and the possibility of incomplete degradation is less likely, since the organisms must extract their entire carbon pool from the single organic compound. In addition, the pressure to select new biochemical abilities via natural mutations is greater in populations in which there are fewer metabolic choices for the community. Complete mineralization may not occur [4], but at least part of the chemical must be mineralized to satisfy the organisms' nutritional requirements.

In this paper we report the use of a continuous culture technique for the selection of a mixed culture of microorganisms that uses bromobenzene as its sole source of carbon and energy. The approach we have used employs mixed populations and single carbon source enrichment cultures. Bromobenzene has proven resistant to mineralization in our hands in direct enrichment studies with bromobenzene as sole source of carbon and energy and chemostat enrichment cultures in which bromobenzene alone was supplied to a mixed microflora grown on benzene. However, when we applied an increasing concentration gradient of bromobenzene and a decreasing benzene concentration gradient to a population of mixed bacteria growing at the expense of benzene, we successfully isolated two bacterial strains capable of using bromobenzene as sole source of 


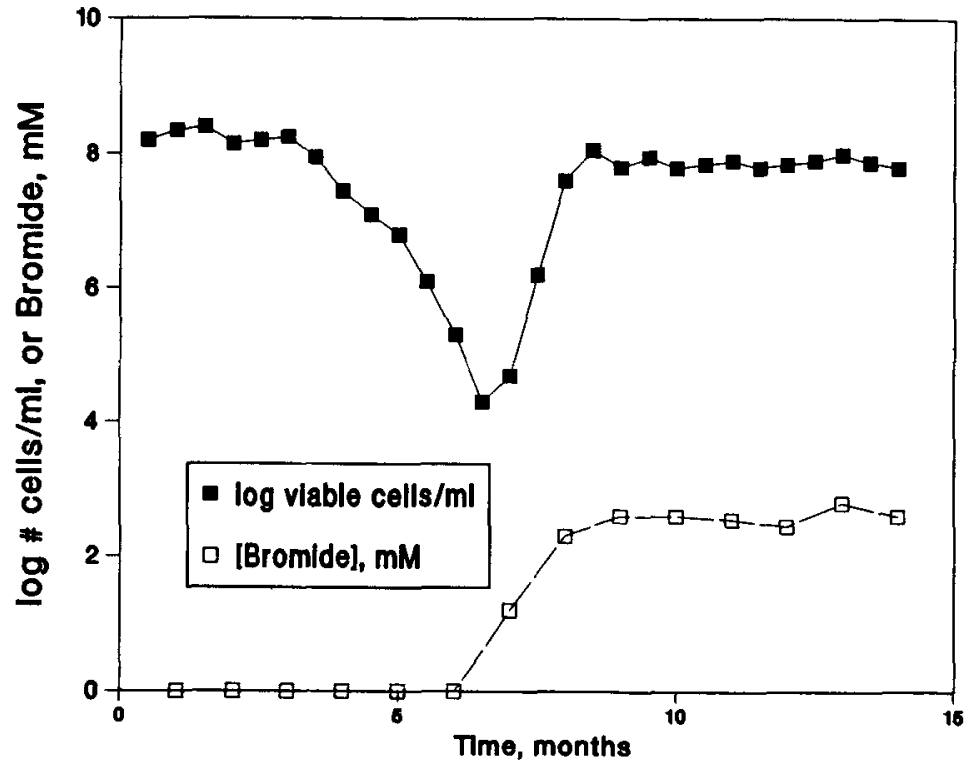

Fig. 1. Growth of the continuous culture. The culture (0-2 months) was grown on benzene. At 2 months the gradient selection began. At approximately 7 months the feed was entirely bromobenzene. carbon and energy. We believe that this technique could have practical use in the selection of strains for toxic chemical waste disposal.

\section{Materials and Methods}

Chemicals. Benzene, iodobenzene, bromobenzene, and chlorobenzene were obtained from the Aldrich Chemical Co. Cinchonidine, strychnine, and brucine sulfate (all $>99.5 \%$ pure) were obtained from Sigma Chemical $\mathrm{Co}$. All chemicals were reagent grade and used without further purification.

Continuous culture. The continuous culture device consisted of the Kem-o-stat (Kontes Glass Co.), culture volume $265 \mathrm{ml}$. The $\mathrm{pH}$ was monitored and remained constant at $\mathrm{pH} 6.6-7.0$ throughout the 14 months of the culture. Growth was at $26^{\circ} \mathrm{C}$, and media were introduced with a peristaltic pump. Except for a small length of silicon tubing in the pump, all connections were glass. The basal medium of Sperl and Hoare [15] was used. The initial population was established with $10 \mathrm{~g}$ wet weight soil suspended in $250 \mathrm{ml}$ of the basal medium. After settling, the supernatant solution was used as an inoculum in the chemostat growth vessel, and air which was passed through liquid benzene was pumped through the air inlet with an aquarium pump. After 10 days of growth on benzene vapors, a thick suspension of microbial growth was observed. At this point the chemostat flow was begun. The medium was the basal medium saturated with benzene (about $8 \mathrm{~m} M$ ). The dilution rate was $0.018 \mathrm{~h}^{-1}$. The first phase of the culture lasted 63 days. Benzene was the growthlimiting factor. The middle stage of the culture was initiated on the 64th day. The device was a simple chemostat with two media reservoirs for production of the benzene/bromobenzene gradient. One reservoir contained 81 of basal medium saturated with bromobenzene (about $3 \mathrm{mM}$ ); the other contained 81 of the basal medium saturated with benzene. The feed was taken from the reservoir, initially containing only benzene-saturated broth. Excess benzene and bromobenzene were always present in these reservoirs to keep the solutions saturated. The two reservoirs were connected at the bottom by a coiled glass tube (tube inner diameter, $<0.5 \mathrm{~mm}$ ) to minimize back mixing. Both reservoirs were stirred slowly to ensure even solutions and mixing. The gradient of increasing bromobenzene/decreasing benzene concentrations lasted 138 days (to about 6.6 months in Fig. 2). The last stage of the culture began on the 213th day. In this stage only one reservoir was used, containing the basal medium saturated with bromobenzene. The continuous culture continued for a total of 14 months.

Assays. All organisms isolated from the first stage of the continuous culture were capable of growth on complex media. Therefore, tryptic soy agar (Difco Co.) was used to determine the number of viable organisms. Pseudomonas agars $P$ and $F$ (Difco Co.) were used in the characterizations of the Pseudomonas isolates. A basal medium was used to determine the number of benzene- or bromobenzene-utilizing microorganisms. Plates (in glass petri dishes) of the basal medium were incubated in a large beaker covered with a watch glass and containing a small beaker of benzene or bromobenzene as the carbon source. During the first 4 months, plate counts from the minimal medium and the basal medium were equivalent. After that only the complex medium was used to determine the number of viable organisms in the culture. Oxygen consumption was determined at $30^{\circ} \mathrm{C}$ with a Yellow Springs (model \#4004) electrode and a mitochondrial chamber (Gilson Medical Electronics). The flagellum stain of Blenden and Goldberg [3] was used. Bromide and iodide were determined by the method of Binkley [2], which is specific for these ions even in the presence of chloride; chloride by the method of Mohr [14]; and catechol by the method of Arnow [1]. Poly- $\beta$-hydroxybutyrate was measured spectrophotometrically [11]. Total bacterial counts were made at 2 -week intervals, and individual members of the community were quantitatively determined every 4 weeks. This was done by picking 1000 colonies at random from the total count plates and noting their colony types. These were then spotted onto Pseudomonas agars $P$ and $F$ plates and basal plates with benzene vapors, as described above. 
Table 1. Microbial constitution of the mixed culture

\begin{tabular}{|c|c|c|c|c|c|c|c|c|c|c|c|c|}
\hline \multirow[b]{2}{*}{ Organism } & \multicolumn{12}{|c|}{$\begin{array}{l}\% \text { Total population } \\
\text { (time, months) }\end{array}$} \\
\hline & 1 & 2 & 3 & 4 & 5 & 6 & 7 & 8 & 9 & 10 & 11 & 12 \\
\hline Nocardia & 62 & 59 & 48 & 39 & 20 & 13 & 8 & $<1$ & 0 & 0 & 0 & 0 \\
\hline Pseudomonas I & 20 & 20 & 28 & 34 & 44 & 56 & 62 & 64 & 62 & 65 & 64 & 65 \\
\hline Pseudomonas II & 15 & 16 & 9 & 6 & $<1$ & 0 & 0 & 0 & 0 & 0 & 0 & 0 \\
\hline Pseudomonas III & 2 & 3 & 9 & 14 & 32 & 30 & 30 & 36 & 38 & 35 & 36 & 35 \\
\hline Pseudomonas IV & 1 & 2 & 6 & 7 & 4 & 1 & 0 & 0 & 0 & 0 & 0 & 0 \\
\hline
\end{tabular}

\section{Results}

The results of our direct enrichment studies showed that benzene is readily and completely mineralized, but its close relatives chlorobenzene, bromobenzene, and iodobenzene are not. We decided to employ the described gradient selection technique with these compounds. After 2, 4, 6, and 8 weeks, the total population of the continuous culture was constant at about $2.2 \times 10^{8}$ viable benzene-utilizing bacteria/ml (Fig. 1). Determination of the individual types of organisms during the first two months indicated five different organisms. There was one Gram-positive organism, tentatively identified as a Nocardia, and four strains with unique colony morphologies of the genus Pseudomonas. The percentages of the total population are shown in Table 1. Pseudomonas I colonies were smooth with a dirty green color, Pseudomonas II colonies were smooth, dirty white with a sunken center, Pseudomonas III colonies were very flat with concentric rings and spreading, and Pseudomonas IV colonies were smooth, small, and heaped dirty white colonies. The Nocardia colonies were small, dry, and wrinkled with a slight pink color. The different colony types were easily identified by eye. Colony morphology was a stable characteristic. The data through the second month showed that the five members of the community remained at relatively constant percentages of the total. Plate counts were conducted twice weekly over this period. Wall growth in this continuous culture never was a serious problem.

After two months, the concentration gradient was begun. It took 4.6 months for the gradient to flow through the system. During this time the concentration of benzene was decreasing from $8 \mathrm{~m} M$ to $0 \mathrm{~m} M$, and the concentration of bromobenzene was increasing from $0 \mathrm{~m} M$ to $3 \mathrm{~m} M$. During this period, the Nocardia and two of the Pseudomonas strains
(II and IV) were washed out of the system, although the Nocardia could be recovered on selective media for Gram-positive organisms (phenylethanol agar plates) at very low levels throughout the gradient period. Later (after eight months) no Nocardia was recoverable. Strains apparently identical with Pseudomonas strains I and III became dominant (Table 1). These two strains eventually established a dual culture in the latter stages of the culture with a constant species proportion of about $2: 1$ (Pseudomonas I: Pseudomonas III). However, Pseudomonas I and Pseudomonas III did not just continue to grow on bromobenzene at the beginning of the gradient, but were initially (3-7 months) washed out at the same rate as the other strains (Fig. 2). What did happen is the appearance in the culture of what appeared to be new biotypes of both Pseudomonas I and III. The first appearance of one of these is at approximately 4.8 months by extrapolation, or 2.8 months after the gradient was begun. From this point, one cell with a maximum specific growth rate constant of $0.021 \mathrm{~h}^{-1}$ would result in the observed growth curve from 7 months onward. This corresponds to a generation time of about $33 \mathrm{~h}$, but because of the presence of increasing bromobenzene and decreasing benzene concentrations, the actual number is difficult to assess. After the establishment of the bromobenzene mixed culture, a maximum growth rate in the chemostat was measured at about $24 \mathrm{~h}$. We have concluded from the data that the Pseudomonas I and III strains that were bromobenzene nonutilizers before the gradient are equivalent to the bromobenzene-utilizing ones after the gradient. The colony morphologies, Gram reaction, cell size, motility, type of flagellation, pigment production on Pseudomonas agars $\mathrm{P}$ and $\mathrm{F}$, cell morphology, and the production of poly- $\beta$-hydroxybutyrate were identical. Other than the different colony morphologies noted above, the only other difference between Pseudomonas I and Pseudo- 


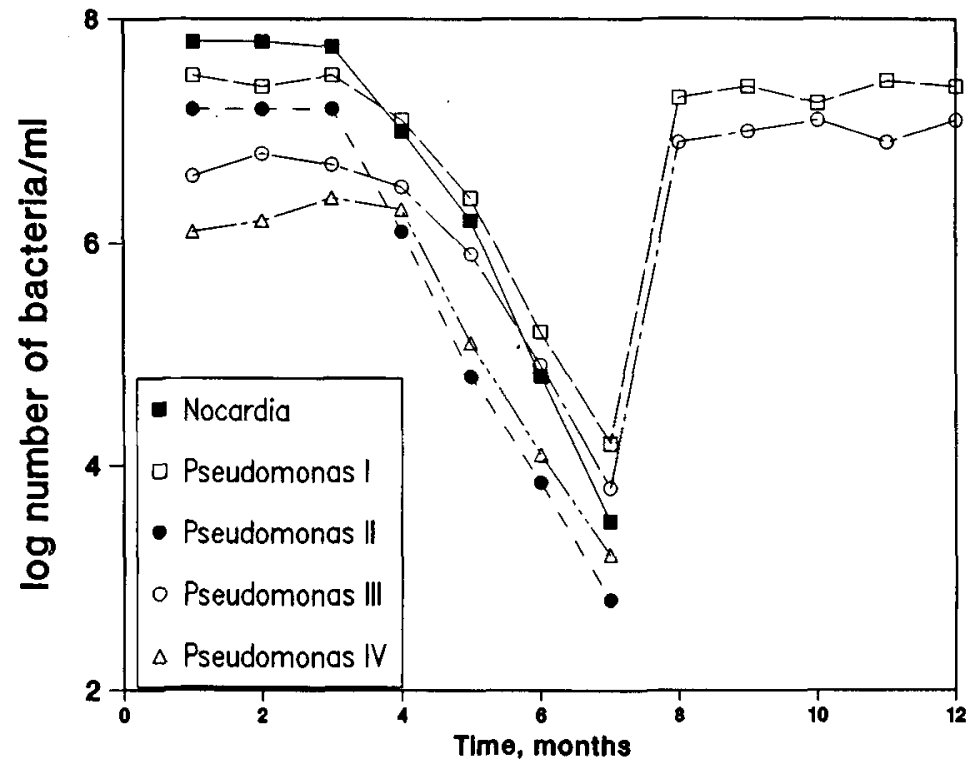

Fig. 2. Effect of the increasing bromobenzene/ decreasing benzene gradient on individual members of the continuous culture. The gradient began at 2 months and finished at approximately 7 months. monas III is that Pseudomonas I often showed more than one polar flagellum per cell, while Pseudomonas III exhibited only one polar flagellum per cell. All of the organisms in the culture after seven months were bromobenzene-utilizing microorganisms.

After 14 months of culture we tested the ability of the culture to utilize chlorobenzene and iodobenzene. Chlorobenzene was used for growth with the concomitant liberation of stoichiometric amounts of chloride $(6.9 \mathrm{mM})$. The same is true for bromobenzene, where $2.8 \mathrm{~m} M$ bromide was released during growth (Fig. 1). However, only $0.1 \mathrm{~m} \mathrm{M}$ iodide was released with iodobenzene, and no visible signs of growth were present. Perhaps iodobenzene is toxic owing to the production of iodine from iodide. Cells of the community (0-2 months) consumed 7.1 moles $\mathrm{O}_{2}$ /mole benzene, but when presented with bromobenzene only 0.9 moles $\mathrm{O}_{2}$ was consumed per mole bromobenzene oxidized. This is consistent with the oxidation of bromobenzene stopping at the level of a catechol derivative, as in Gibson et al. [5]. These authors showed that bromobenzene was metabolized to 3-bromocatechol by benzene-grown cells of Pseudomonas putida, but complete dissimilation did not occur. In our experiments, almost equimolar amounts of a catechol were produced during the oxidation of bromobenzene by benzenegrown, bromobenzene-nonutilizing cells. However, after the 7 -month point, 6.8 moles of $\mathrm{O}_{2} /$ mole of bromobenzene oxidized were consumed, and little catechol was detected. The stoichiometric libera- tion of the halide (bromide or chloride) and the ability to support a high population density (about $10^{8}$ cells $/ \mathrm{ml}$ ) as well as the oxygen consumption data indicate that bromo- or chlorobenzene was completely dissimilated. These cells continued to be able to mineralize benzene at equivalent rates. There was no other available carbon source after the gradient was finished.

\section{Discussion}

We have shown a way of isolating microorganisms capable of completely degrading a normally nonmetabolized, toxic compound. The use of the chemostat allows a slow selection, with steady pressure on the population. Certainly the technique would not be successful for all compounds, and compounds that are biochemically so inert such as chlordane or DDT will probably not be successful candidates for this method [12]. However, compounds of intermediate recalcitrance might well become more readily treated microbiologically, thus adding to the methods available now for the elimination of toxic chemicals from spills and dumps.

Although the data are not unequivocal, they support the conclusion that the Pseudomonas strains developed here arose from mutation of the Pseudomonas I and III strains. About $3 \times 10^{12}$ bacteria were produced in the chemostat before the first bromobenzene-utilizing organism appeared. A mutational frequency of $10^{-6}$ would imply that this appearance was a double mutation, but a mutational 
frequency of $10^{-12}$ is not out of reality. There is also the problem that two different Pseudomonas strains became bromobenzene utilizers. Either the mutation(s) happened in both, or there was some genetic exchange, which is quite possible [6]. When $10^{10}$ actively growing washed cells from the benzene portion of the continuous culture were put in a medium with bromobenzene, they did not grow. Therefore, the mutational frequency to the bromobenzene-utilizing phenotype is probably less than $10^{-10}$. There is the possibility that there always existed a very small number of microbes in the chemostat which could use bromobenzene, but which evaded our sampling and enrichment efforts. We feel that our enrichment cultures would have found these, but it is impossible to completely eliminate this possibility. If there were enzyme evolution in these experiments, whether one or two mutations, we do not know which enzymes were altered. It seems likely from the accumulation of catechol and the work of Gibson et al. [5] that the catechol oxygenase is a likely candidate, but we have no definitive evidence for this. Enzyme evolution is known to occur in chemostats without apparent pressure [13], so the changeable nature of the microbial genomes is also an important factor to consider.

\section{Literature Cited}

1. Arnow LE (1937) Colorimetric determination of the components of 3,4-dihydroxyphenylalanine-tyrosine mixtures. J Biol Chem 118:531-537
2. Binkley F (1947) A colorimetric reaction of chloride ion. J Biol Chem 148:403-406

3. Blenden DC, Goldberg HS (1965) Silver impregnation stain for Leptospira and flagella. J Bacteriol 89:899-900

4. Daughton CG, Hsieh DPH (1977) Parathion utilization by bacterial symbionts in a chemostat. Appl Environ Microbiol $34: 175-184$

5. Gibson DT, Koch JR, Schuld CL, Kallio RE (1968) Oxidative degradation of aromatic hydrocarbons by microorganisms. II. Metabolism of halogenated aromatic hydrocarbons. Biochemistry 7:3795-3802

6. Gunsalus IC, Marshall VP (1972) Monoterpene dissimilation: chemical and genetic models. CRC Crit Rev Microbiol 1:291-310

7. Hallas LE, Alexander M (1983) Microbial transformations of nitroaromatic compounds in sewage effluent. Appl Environ Microbiol 45:1234-1241

8. Horvath RS (1972) Microbial cometabolism and the degradation of organic compounds in nature. Bacteriol Rev 36:146155

9. Jacobson SN, O'Mara NL, Alexander M (1980) Evidence for cometabolism in sewage. Appl Environ Microbiol 40:917921

10. Katan J, Fuhremann TW, Lichtenstein EP (1976) Binding of ${ }^{14} \mathrm{C}$-parathion in soil: a reassessment of pesticide residues. Science 193:891-894

11. Law JH, Slepecky RA (1961) Assay of poly- $\beta$-hydroxybutyric acid. J Bacteriol 82:33-42

12. Matsumara F, Bousch GM (1972) In: McLaren AD, Skujins J (eds) Soil Biochemistry, vol. 2. New York: Marcel Dekker, Inc, pp 320-336

13. Senior E, Bull AT, Slater JH (1976) Enzyme evolution in a microbial community growing on the herbicide Dalapon. Nature 263:476-479

14. Skoog DA, West DM (1976) Fundamentals of analytical chemistry. New York: Holt, Rinehart and Winston

15. Sperl GT, Hoare DS (1971) Denitrification with methanol: a selective enrichment for Hyphomicrobium species. J Bacteriol 108:733-736 